\title{
Prevalence and determinants of asphyxia neonatorum among live births at Debre Tabor General Hospital, North Central Ethiopia: a cross-sectional study
}

\author{
Wubet Alebachew Bayih ${ }^{1}$, Tadesse Gashaw Tezera ${ }^{1}$, Abebaw Yeshambel Alemu ${ }^{1}$, Demeke Mesfin Belay ${ }^{1}$, \\ Habtamu Shimelis Hailemeskel ${ }^{1}$, Metadel Yibeltal Ayalew ${ }^{2}$
}

\begin{abstract}
1. College of Health Sciences, Debre Tabor University, P.O.BOX. 272, Debre Tabor, Ethiopia.
2. Tibebe Ghion Specialized Hospital, Bahir Dar University, Bahir Dar, Ethiopia.
\end{abstract}

Authors' affiliation

Wubet Alebachew Bayih: wubetalebachew@gmail.com; Tadesse Gashaw Tarekegn: tadessegashaw37@gmail.com; Habtamu Shimelis Hailemeskel:Habtamushimels21@gmail.com; Abebaw Yeshambel Alemu: yeshambelabebaw@gmail.com; Demeke Mesfin Belay:demekemesfin65@yahoo.com

\begin{abstract}
Background: More than one third of the neonatal deaths at Neonatal Intensive Care Unit of Debre Tabor General Hospital (DTGH) are attributable to birth asphyxia. Most of these neonates are referred from maternity ward of the hospital. However, there is no recent evidence on the prevalence and specific determinants of birth asphyxia at DTGH. Besides, public health importance of factors like birth spacing weren't addressed in the prior studies.

Methods: A cross sectional study was conducted on a sample of 240 newborns at delivery ward. The collected data were cleaned, coded and entered into Epi -data version 4.2 and exported to Stata version 14. Binary logistic regression model was considered and statistical significance was declared at $\mathrm{P}<0.05$ using adjusted odds ratio.

Results: The prevalence of asphyxia neonatorum was $6.7 \%$ based on the fifth minute APGAR score. From multi-variable logistic regression analysis, antenatal obstetric complications (AOR $=2.63,95 \% \mathrm{CI}: 3.75,14.29)$, fetal malpresentation (AOR $=3.17,95 \% \mathrm{CI}: 1.21,15.20)$, premature rupture of fetal membranes (AOR $=6.56,95 \% \mathrm{CI}: 3.48,18.12)$ and meconium stained amniotic fluid (AOR $=2.73,95 \%$ CI: $1.76,14.59)$ were significant predictors.

Conclusion: The prevalence of fifth minute asphyxia neonatorum was relatively low. Fortunately, its predictors are modifiable. Thus, we can mitigate the problem even with our limited resources such as enhancing the existing efforts of antenatal and intra-partum care, which could help early detection and management of any obstetric and neonatal health abnormality." Keywords: Birth asphyxia; asphyxia neonatorum; prevalence; determinants; Ethiopia.

DOI: https://dx.doi.org/10.4314/ahs.v21i1.49

Cite as: Bayih WA, Tezera TG, Alemu AY, Belay DM, Hailemeskel HS, Ayalew MY. Prevalence and determinants of asphyxia neonatorum among live births at Debre Tabor General Hospital, North Central Ethiopia: a cross-sectional study. Afri Health Sci. 2021;21(1):385-96. bttps:/ / dx.doi.org/10.4314/abs.v21i1.49
\end{abstract}

\section{Introduction}

Asphyxia neonatorum or birth asphyxia is defined as "failure to initiate and sustain spontaneous breathing at birth $^{1,2}$. It can also be defined as placental or pulmonary gas exchange impairment leading to hypoxemia and hypercarbia with a resultant effect of metabolic acidosis (evidenced by low umbilical cord $\mathrm{pH}<7$ ), low APGAR score less than 3 at $10^{\text {th }}$ minute and abnormal muscle

\section{Corresponding author:}

Metadel Yibeltal Ayalew,

Tibebe Ghion Specialized Hospital,

Bahir Dar University, Bahir Dar, Ethiopia.

Email: metadelyibeltal2@gmail.com tone ${ }^{3-6}$. Besides, the American College of Obstetrics and Gynecology (ACOG) and American Academy of Pediatrics (AAP) defined birth asphyxia based on three (3) factors: metabolic or mixed acidemia determined from umbilical cord blood samples $(\mathrm{pH}<7)$, APGAR score of less than 3 for longer than five minute and multisystem organ dysfunction ${ }^{5}$. The most valid diagnosis of birth asphyxia is made from the combination of three parameters. These are APGAR score less than 7 , results of arterial blood gas analyses $\left(\mathrm{PO}_{2}\right.$ and $\left.\mathrm{PCO}_{2}\right)$ and immediate newborns' umblical cord blood $\mathrm{pH}$ of $<7^{6}$. However, in resource poor countries like Ethiopia where there is infeasibility of the latter two parameters, the parameter of Apgar score is used to diagnose birth asphyxia, evaluated in the fifth minute of life, with work is properly cited. 
scores ranging from zero to ten. Thus, a diagnosis of birth asphyxia can be made when neonatal fifth minute Apgar score is less than ${ }^{7}$. Apgar score values of four to seven indicate moderate birth asphyxia whereas severe asphyxia is between zero and three ${ }^{7,54}$.

Asphyxia neonatorum endangers neonatal survival. Severe degrees of birth asphyxia can cause severe neonatal multiorgan damage resulting in brain damage, lung dysfunction, cardiomyopathy, renal failure, hepatic failure and necrotizing enterocolitis ${ }^{7-27}$. From these damages, brain damage is of the greatest concern becausvictim newborns are less likely to survive and even the survivors are more likely to have long-term neurological morbidities like cerebral palsy, permanent seizure disorder, intellectual incompetence and motor deficits, thereby leaving the survivors to be lifetime family burden. This in turn has raised the demand for costly technological care of asphyxiated newborns though the treatment outcome is often poor ${ }^{3-5}$.

Asphyxia neonatorum can be caused by factors related to the antepartum, intra partum or post partum peri$\mathrm{od}^{28-49}$. However, quality of intrapartum care during labor and delivery has been recognized as the single most important predictor of the overall morbidity and mortality from asphyxia neonatorum ${ }^{32,34,36-49}$. More specifically, factors like antenatal obstetric complications 17,20 , 40, 42, 49,51-56, parity ${ }^{19,42}$, multiple births ${ }^{22}$, gestational age $<37$ or $>41$ weeks ${ }^{22,42}$, low birth weight ${ }^{18,40,41,42}$, premature rupture of membranes ${ }^{20,44,45,48}$, prolonged labor 18, 19, 41,42 and fetal distress 40,41, 48, 49 have already been identified to be among the risk factors of asphyxia neonatorum.

The Ethiopian neonatal mortality rate (NMR) is 29/1000 live births whilst Amhara region, a region of our study area, comprises the highest burden of the national NMR (47/1000 live births) ${ }^{16}$. Literature shows asphyxia neonatorum is a universal public health problem with varied significance country wise $9,15,16$. For example, $31.6 \%$ of the Ethiopian neonatal mortality is attributed to asphyxia neonatorum ${ }^{24}$.

A significant number of neonatal deaths (33.3\%) at Neonatal Intensive Care Unit (NICU) of Debre Tabor General Hospital (DTGH) are attributable to birth asphyxia. Most of these neonates are referred from maternity ward of the hospital. However, there is no recent evidence on the proportion and specific determinants of birth asphyxia at DTGH ${ }^{50,53}$. Moreover, the role of some important factors like birth spacing and birth weight for gestational age weren't addressed in the prior studies. Therefore, this study was aimed at deter- mining the prevalence and specific predictors of birth asphyxia among newborns delivered at maternity ward of Debre Tabor General Hospital, Debre Tabor Town, South Gonder Zone, North Central Ethiopia,which is helpful to make interventions appropriate to the context of DTGH.

\section{Methods}

\section{Study setting, period and design}

A hospital-based quantitative cross sectional study was conducted from January first to April first 2019 at delivery ward of Debre Tabor General Hospital. The hospital is located in Debre Tabor town, $666 \mathrm{~km}$ far from Addis Ababa. It serves about 3,840 deliveries annually ${ }^{50}$.

\section{Study participants}

Mothers who gave live birth after 28 weeks of gestational age were screened for eligibility. Newborns of unknown gestational age at birth were excluded. Furthermore, newborns with malformations incompatible to life such as hydrops and cyanotic congenital heart defects were excluded as these newborns have already been predisposed for asphyxia.

\section{Sample size determination and sampling tech- nique}

A total sample of 240 mother-newborn dyads was obtained using single population proportion formula; considering a confidence level of 95\%, marginal error of $5 \%$, a reasonable estimate for the proportion of asphyxia neonatorum from a previous study $(\mathrm{p}=0.829) 38$ and adding a none response rate of $10 \%$. The average monthly delivery rate of DTGH was 320 as per DTGH'S last quarter report of 2018.Then, to ensure representativeness of the calculated sample size $(n=240)$, every fourth eligible mother newborn dyad was selected over 3 months at maternity ward of DTGH.

\section{Data collection procedure}

A pretested interviewer based questionnaire was used to collect primary data on maternal socio-demographic and ante partum related factors. For twin births, every mother was asked only once about socio-demographic and antenatal factors of her twin babies because twin neonates share similar socio-demography and antenatal history. Furthermore, a pretested structured checklist was employed to abstract secondary data from maternal chart on intra-partum (induction and/augmentation, fetal distress, fetal presentation at birth, mode of delivery, fetal distress, time of membrane rupture, duration of labor, color of amniotic fluid) and neonatal related 
factors (sex, birth weight, gestational age at birth and birth weight to gestational ag). For twin births, every mother's chart was reviewed twice for the aforementioned intrapartum and neonatal related characteristics due to variation of these factors between twin neonates.

Fifth minute APGAR score was collected from direct observation by six trained BSc graduating class midwifery students both during day and night shifts in the delivery ward and operation room, which we got it feasible. Apgar score was measured using five components (Skin color / oxygenation; totally pinkish body ( 2 points), body pink but bluish extremities (1 point), totally blue or pale body (0 point)); (Pulse or heart rate ; $\geq 100$ beats/minute ( 2 points), 100 (1 point), absent ( 0 point)); (Grimace or reflex irritability to stimuli; Crying or coughing or sneezing ( 2 points), grimacing (1 point), no response at all ( 0 point $)$ );(Activity or muscle tone and movement; well flexed or active motion ( 2 points), some flexion of extremities (1 point), flaccid or limp (0 point)) and (Respiration or breathing effort or lung maturity; (Good cry or regular breathing (2 points), weak or irregular and $<30$ breath/minute (1 point), absent or apnea ( 0 point). Then, the score of each component was summed up by the data collectors and the information was documented on an Apgar score card (additional file 1).

The diagnosis of birth asphyxia was made based on fifth minute APGAR score..The diagnoses of birth asphyxia were further confirmed through medical interns' consultation of obstetricians and pediatricians to deal with its severity and management.

The questionnaire and checklist were adapted from Ethiopian and other African studies 17-19, 20, 22, 40-42. The questionnaire was first prepared in English and translated to Amharic (local language to conduct interview) and retranslated to English to check for consistency. Before the actual data collection, pretest was done using 12 eligible mother- newborn dyads ( $5 \%$ of sample size) at the same hospital a week before data collection. One day training was first provided for data collectors and supervisors on the process of data collection.

\section{Operational definitions}

Birth asphyxia: A newborn was considered to have birth asphyxia when its fifth minute APGAR score was $<7^{7,54}$.

\section{Prolonged labor}

Prolonged labor (second stage): For nulliparous mothers, if labor exceeds 3 hours with provision of regional anesthesia, or 2 hours without regional anesthesia. For multiparous mothers, if labor exceeds 2 hours with regional anesthesia or 1 hour in the absence of regional anesthesia. We have assessed this in numbers. ${ }^{7}$. Prolonged labor was collected from the maternal chart. Premature rupture of membrane was diagnosed when fetal chorio-amnionic membranes rupture at any time before the onset of true labor ${ }^{7}$.

\section{Statistical analysis}

The collected data were coded, cleaned, edited and double entered into epidata version 4.2 after which it was exported to STATA version 14. Frequencies, proportion, summary statistics and cross tabulation were used to describe the study population. Using binary logistic regression model, bivariable analysis was first carried out to identify candidate variables $(\mathrm{P}<0.25)$ for multivariable analysis. Then, multivariable logistic regression analysis was performed to investigate statistically significant predictors of asphyxia neonatorum by adjusting for possible confounders. Finally, variables $(\mathrm{P}<0.05)$ were reported as statistically significant using AOR with $95 \% \mathrm{CI}$.

\section{Results}

\section{Socio -demographic factors}

In this study, the overall 240 respondents were involved thereby making a response rate of $100 \%$. More than half of the respondents, $145(60.4 \%)$ were rural residents. Moreover, 221(92.9\%) of the respondents were married and concerning their educational status, about one third of them $(32.4 \%)$ were unable to read and write. The mean maternal age was $28.70(\mathrm{SD}= \pm 5.86)$ years of whom about three quarters, $185(77 \%)$ were in the age group of ( $>34$ years). Nearly half of the mothers, $125(52.5 \%)$ have less than two years of birth spacing for the index newborn. Besides, about one quarter, $53(22.1 \%)$ of the mothers had history of adverse pregnancy outcome of which prematurity 23(43.4\%) accounted for the highest proportion (Table 1). 
Table 1: Socio-demographic factors of mothers who gave live birth at Debre Tabor General Hospital, $2019(\mathrm{n}=240)$.

\begin{tabular}{|c|c|c|c|}
\hline Factor & Response & $\mathrm{n}$ & $\%$ \\
\hline \multirow[t]{2}{*}{ Residence } & Rural & 145 & $60.4 \%$ \\
\hline & Urban & 95 & $39.6 \%$ \\
\hline \multirow[t]{3}{*}{ Age } & $15-19$ & 15 & $6.3 \%$ \\
\hline & $20-34$ & 40 & $16.7 \%$ \\
\hline & $>34$ & 185 & $77.0 \%$ \\
\hline \multirow[t]{3}{*}{ Marital status } & Married & 221 & $92.1 \%$ \\
\hline & Widowed & 14 & $5.8 \%$ \\
\hline & Separated & 5 & $2.1 \%$ \\
\hline \multirow[t]{2}{*}{ Religion } & Orthodox & 221 & $92.1 \%$ \\
\hline & Muslim & 19 & $7.9 \%$ \\
\hline \multirow[t]{4}{*}{ Occupation } & House wife & 141 & $58.8 \%$ \\
\hline & Governmental Employee & 41 & $17.0 \%$ \\
\hline & Merchant & 16 & $6.7 \%$ \\
\hline & Daily Labor & 42 & $17.5 \%$ \\
\hline \multirow[t]{5}{*}{ Educational Status } & Unable to read and write & 77 & $32.1 \%$ \\
\hline & $\begin{array}{l}\text { No formal education but can } \\
\text { read and write }\end{array}$ & 78 & $32.5 \%$ \\
\hline & Primary education (1-8) & 42 & $17.5 \%$ \\
\hline & Secondary education (9-12) & 19 & $7.9 \%$ \\
\hline & College or University & 24 & $10 \%$ \\
\hline \multirow[t]{2}{*}{ Gravidity } & $<3$ & 72 & 30 \\
\hline & $\geq 3$ & 168 & 70 \\
\hline \multirow[t]{2}{*}{ Parity } & Primiparous & 61 & 25.4 \\
\hline & Multiparous & 179 & 74.6 \\
\hline \multirow{2}{*}{ Birth spacing (Years) } & $<2$ & 125 & 52.1 \\
\hline & $\geq 2$ & 115 & 47.9 \\
\hline \multirow{2}{*}{$\begin{array}{l}\text { History of adverse pregnancy } \\
\text { outcome }\end{array}$} & Yes & 53 & 22.1 \\
\hline & No & 187 & 77.9 \\
\hline \multirow{5}{*}{$\begin{array}{l}\text { Type of adverse pregnancy } \\
\text { outcome } ?(\mathbf{n}=\mathbf{5 3})\end{array}$} & Abortion & 7 & 13.2 \\
\hline & Intrauterine fetal death & 6 & 11.3 \\
\hline & Still birth & 9 & 17.0 \\
\hline & Preterm & 23 & 43.4 \\
\hline & Neonatal death & 8 & 15.1 \\
\hline
\end{tabular}

\section{Ante partum related factors}

Nearly all the mothers, 233(95.8\%) had attended antenatal care at public hospitals $(59.7 \%)$ and public health center $(40.3 \%)$. However, it was only about half of the mothers $(52.6 \%)$ who had four and above antenatal care visits. About one tenth of the mothers (11.25\%) ever used substance during their gestation of which ever al- cohol users accounted for the most majority (70.4\%). In the antenatal period, $87(36.3 \%)$ mothers were found to have obstetric complications. From these complications, preeclampsia/eclampsia accounted for the highest percentage $(37.9 \%)$ followed by antepartum hemorrhage $(23.0 \%)$, anemia $(21.8 \%)$, infections $(11.5 \%)$ and gestational diabetes $(5.7 \%)$ (Table 2$)$. 
Table 2: Factors related to the ante partum period among mothers who gave live birth at Debre Tabor General Hospital, $2019(n=240)$.

\begin{tabular}{|c|c|c|c|}
\hline Factor & Response & $\mathrm{N}$ & $\%$ \\
\hline \multirow[t]{2}{*}{$\mathrm{ANC}$} & Yes & 233 & 95.8 \\
\hline & No & 7 & 4.2 \\
\hline \multirow{2}{*}{$\begin{array}{l}\text { Number of ANC visits } \\
(\mathbf{n}=\mathbf{2 3 3})\end{array}$} & $<4$ & 112 & 48.1 \\
\hline & $\geq 4$ & 121 & 51.9 \\
\hline \multirow{2}{*}{$\begin{array}{l}\text { Obstetric complication during } \\
\text { pregnancy }\end{array}$} & Yes & 87 & 36.3 \\
\hline & No & 153 & 63.8 \\
\hline \multirow[t]{5}{*}{ Type of complication $(\mathbf{n}=\mathbf{8 7})$} & Preeclampsia/eclampsia & 33 & 37.9 \\
\hline & Antepartum hemorrhage & 20 & 23.0 \\
\hline & Anemia & 19 & 21.8 \\
\hline & Infections & 10 & 11.5 \\
\hline & Gestational diabetes & 5 & 5.7 \\
\hline \multirow{2}{*}{$\begin{array}{l}\text { Ever used substance during } \\
\text { pregnancy }\end{array}$} & Yes & 27 & 11.25 \\
\hline & No & 213 & 88.75 \\
\hline \multirow{3}{*}{$\begin{array}{l}\text { Type of substance ever used } \\
\text { during pregnancy }(\mathbf{n}=\mathbf{2 7})\end{array}$} & Alcohol & 19 & 70.4 \\
\hline & Khat & 6 & 22.2 \\
\hline & Cigarette & 2 & 7.4 \\
\hline
\end{tabular}

\section{Intra partum related factors}

Of the total respondent mothers, $144(60 \%)$ had spontaneous labor onset. Majority of the mothers 188(78.3\%) had intrapartum rupture of fetal membranes whereas premature rupture of membrane was reported among $52(21.7 \%)$ mothers. Following membrane rupture, meconium stained amniotic fluid was observed among $97(40.4 \%)$ mothers and similar report was obtained to the presence of fetal distress 98 (40.8\%). At labor, about one third of the fetuses $73(30.4 \%)$ were malpresented and nearly equal number of fetuses were delivered by cesarean section $71(29.6 \%)$ (Table 3).

Table 3: Factors related to the intrapartum period among mothers who gave live birth at Debre Tabor General Hospital, $2019(n=240)$.

\begin{tabular}{|c|c|c|c|}
\hline Factor & Response & $\mathrm{N}$ & $\%$ \\
\hline \multirow[t]{2}{*}{ Fetal presentation } & Vertex & 167 & 69.6 \\
\hline & Malpresentation & 73 & 30.4 \\
\hline \multirow[t]{2}{*}{ Mal-presentation type $(n=73)$} & Breech & 52 & 71.2 \\
\hline & Face & 21 & 28.8 \\
\hline \multirow[t]{3}{*}{ Labor type } & Spontaneous onset & 144 & 60 \\
\hline & Induced & 34 & 14.2 \\
\hline & Augmented & 62 & 25.8 \\
\hline \multirow[t]{3}{*}{ Labor duration } & Normal & 177 & 73.8 \\
\hline & Prolonged & 44 & 18.3 \\
\hline & Precipitated & 19 & 7.9 \\
\hline \multirow[t]{2}{*}{ Time of membrane rupture } & PROM & 52 & 21.7 \\
\hline & Intrapartum & 188 & 78.3 \\
\hline \multirow{2}{*}{$\begin{array}{l}\text { Duration of PROM until delivery } \\
\text { occurs } \boldsymbol{n}=\mathbf{5 2}\end{array}$} & Normal & 39 & 75 \\
\hline & Prolonged & 13 & 25 \\
\hline \multirow[t]{2}{*}{ Color of amniotic fluid } & Meconium stained & 97 & 40.4 \\
\hline & Clear & 143 & 59.6 \\
\hline \multirow[t]{2}{*}{ Fetal distress } & Yes & 98 & 40.8 \\
\hline & No & 142 & 59.2 \\
\hline \multirow[t]{3}{*}{ Mode of delivery } & SVD & 139 & 57.9 \\
\hline & $\mathrm{C} / \mathrm{S}$ & 71 & 29.6 \\
\hline & Instrumental & 30 & 12.5 \\
\hline \multirow[t]{4}{*}{ Birth attendant } & Midwife & 142 & 59.2 \\
\hline & Emergency surgeon & 48 & 20 \\
\hline & Obstetrician & 23 & 9.6 \\
\hline & Medical intern & 17 & 7.1 \\
\hline
\end{tabular}




\section{Newborn related characteristics}

More than half of the newborns, 132 (55\%) were males. There were 34 twin newborns. The mean gestational age at birth was $38.3( \pm 2.3)$ weeks and majority of the newborns $(64.2 \%)$ were term. Moreover, the mean birth weight was $2674.3( \pm 1484.2)$ grams and about one third of the newborns $73(30.4 \%)$ had low birth weight. When birth weight was considered against the corresponding gestational age at birth, about one fifth of the total newborns $53(22.1 \%)$ had birth weight inappropriate for their gestational age at birth. Of those $53(22.1 \%)$ newborns with inappropriate birth weight for gestational age, $24(10 \%)$ were small for their gestational age whereas the remaining 29 newborns (12.1\%) had large birth weight for their corresponding gestational age (Table 4).

Table 4: Newborn related characteristics at Debre Tabor General Hospital, 2019 ( $\mathrm{n}=240)$.

\begin{tabular}{llll}
\hline Factor & Response & $\mathrm{n}$ & $\%$ \\
Sex & Male & 132 & 55 \\
\multirow{3}{*}{ Birth outcome } & Female & 108 & 45 \\
\multirow{3}{*}{ Birth weight } & Singleton & 206 & 85.8 \\
& Twin & 34 & 14.2 \\
Gestational age at birth & $<2500$ & 73 & 30.4 \\
& $\geq 2500$ & 167 & 69.6 \\
\multirow{3}{*}{$\begin{array}{l}\text { Birth weight for Gestational age } \\
\text { at birth }\end{array}$} & Preterm & 71 & 29.6 \\
& Term & 154 & 64.2 \\
& Post term & 15 & 6.3 \\
& Appropriate for gestational age & 187 & 77.9 \\
& Small for gestational age & 24 & 10.0 \\
& Large for gestational age & 29 & 12.1
\end{tabular}

Proportion Prevalence of asphyxia neonatorum be $16(6.7 \%)$ (Fig 1). All cases of the birth asphyxia The prevalence of asphyxia neonatorum was found to were of moderate severity.

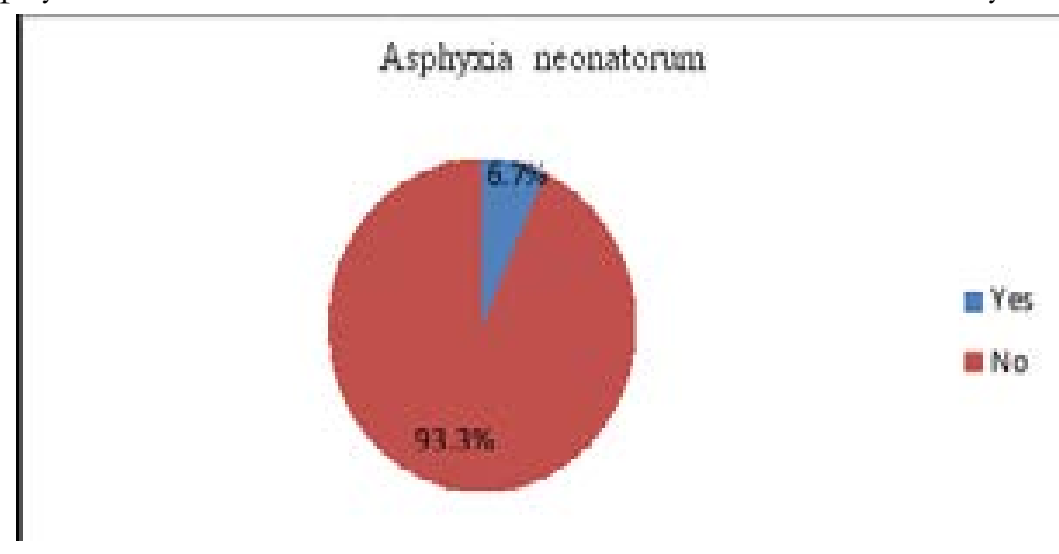

Figure 1: Prevalence of asphyxia neonatorum among live births at DTGH (n=240), 2019.

Besides, first minute APGAR score showed that 99 $(41.3 \%)$ neonates had less than 7 APGAR score. Most of these neonates, $96(40.0 \%)$ had

\section{Determinants of asphyxia neonatorum}

The bivariable logistic regression analysis showed that antenatal obstetric complications, fetal malpresentation, premature rupture of fetal membranes, meconium stained amniotic fluid, fetal distress, mode of delivery and birth spacing were crudely associated with asphyxia neonatorum. However, after statistical adjustments for the likely effects of confounding variables, only four of the aforementioned factors namely antenatal obstetric complications, fetal malpresentation, premature rupture of fetal membranes and meconium stained amniotic fluid, were significant predictors of asphyxia. In this study, the width interval for three of the adjusted point estimates (premature rupture of membrane, fetal malpresentation and meconium stained amniotic fluid) is somewhat wider, thereby indicating a relatively less precise measurement of these factors. However, the width interval for the CIs of these factors is less than 17 , which is in the recommended gap as shown in table 5 given below. 
Table 5: Precision of the point estimates on the significant determinants of asphyxia neonatorum among live births at Debre Tabor General Hospital, North Central Ethiopia, $2019 \mathrm{n}=240$.

\begin{tabular}{|l|l|l|l|l|}
\hline Factor & $\begin{array}{l}\text { Adjusted effect } \\
\text { size } \\
\text { estimate (AOR) }\end{array}$ & $\begin{array}{l}\text { Lower } \\
\text { bound }\end{array}$ & $\begin{array}{l}\text { Upper } \\
\text { bound }\end{array}$ & $\begin{array}{l}\text { Width of CIs (i.e. } \\
\begin{array}{l}\text { Upper bound } \\
\text { minus lower } \\
\text { bound) }\end{array}\end{array}$ \\
\hline Premature rupture of membrane & 6.56 & 3.48 & 18.12 & 14.64 \\
\hline Fetal mal presentation presentation & 3.17 & 1.21 & 15.20 & 13.99 \\
\hline Meconium stained amniotic fluid & 2.73 & 1.76 & 14.59 & 12.83 \\
\hline Antenatal obstetric complications & 2.63 & 3.75 & 14.29 & 10.54 \\
\hline
\end{tabular}

The amendment to the original manuscript is included in the revised version manuscript as shown by the tracked insertions under the determinants of asphyxia neonatorum and limitation of the study on pages 9,12 and 13.

The likelihood of developing birth asphyxia among neonates born to mothers with antenatal obstetric complications was 2.63 times (AOR $=2.63,95 \%$ CI: 3.75, 14.29) more when compared to their counter-parts. Neonates born with fetal malpresentation had 3.17 times
$(\mathrm{AOR}=3.17,95 \% \mathrm{CI}: 1.21,15.20)$ more likelihood of being asphyxiated at birth as compared to those of vertex presentations. Neonates born to mothers with premature rupture of fetal membranes were 6.56 times $(\mathrm{AOR}=6.56,95 \% \mathrm{CI}: 3.48,18.12)$ more prone to be asphyxiated at birth when compared to those with intrapartum rupture of membranes. Similarly, neonates born to mothers having meconium stained amniotic fluid were 2.73 times (AOR $=2.73,95 \% \mathrm{CI}: 1.76$, 14.59) as likely to have birth asphyxia as compared to those born without being meconium stained.

Table 6: Bivariable and multivariable logistic regression analysis of factors associated with asphyxia neonatorum among live births at Debre Tabor General Hospital, North Central Ethiopia, 2019 ( $n=240)$.

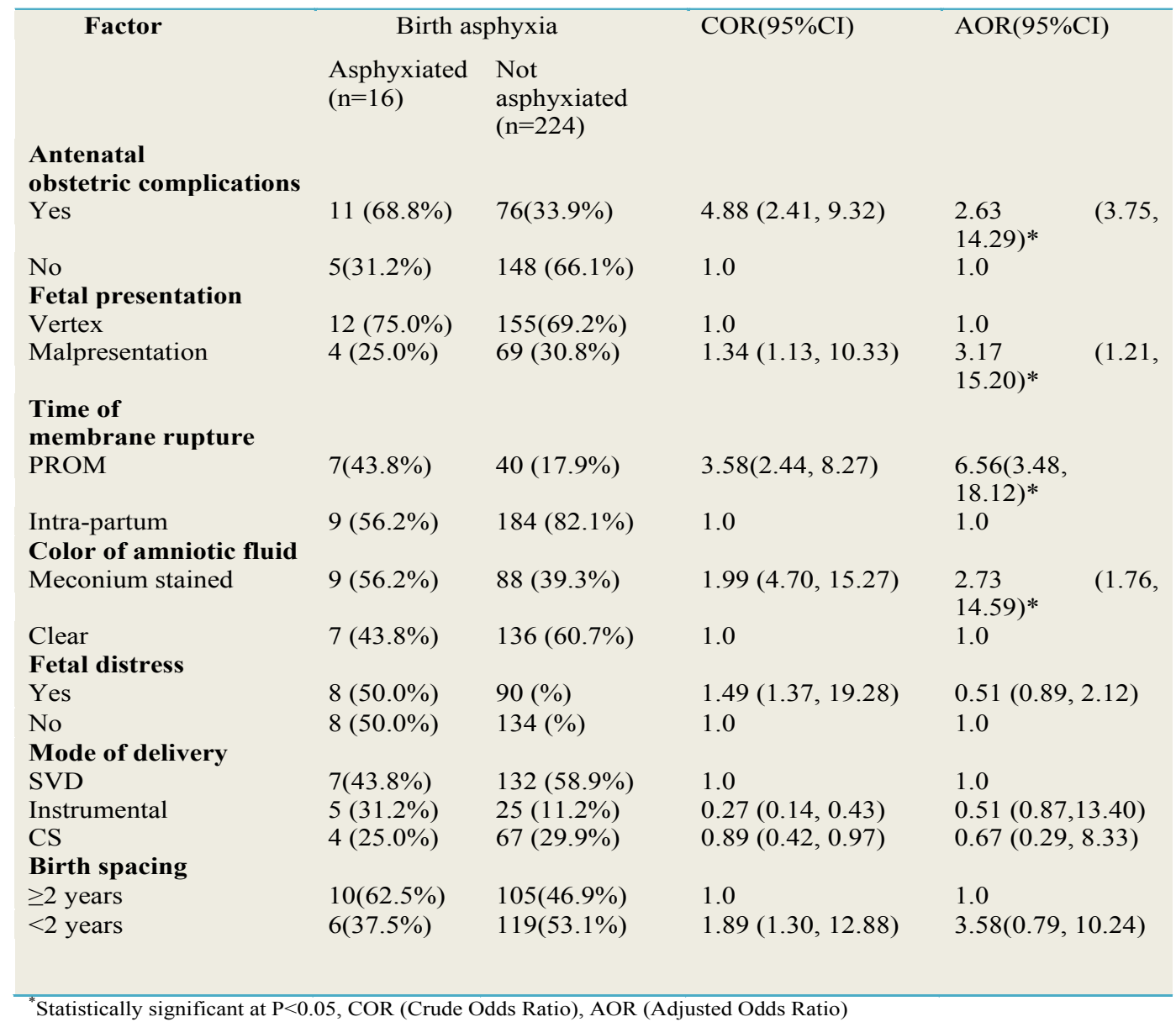




\section{Discussion}

Hypoxic-ischemic damage of neonatal vital organs mainly during the intrapartum period is the leading cause of mortality from asphyxia neonatorum. Thus, the quality of obstetric care at birth is crucial to reduce the overall newborn mortality and its long-term consequences ${ }^{1,2,4,6}$. In this study, it was tried to identify the predictors of asphyxia neonatorum among live births at Debre Tabor General Hospital. Antenatal obstetric complications, fetal malpresentation, premature rupture of fetal membranes, prolonged labor, meconium stained amniotic fluid, fetal distress and CS delivery were found to be significant predictors of asphyxia neonatorum.

"From this study, the prevalence of birth asphyxia was $6.7 \%$, which was lower than a study in Iran $(58.8 \%)^{21}$. This may be due to differences in sample size and study setting. Besides, most importantly, difference in case definition might have played contribution for the variation; for example, our study was based on only fifth minute APGAR score less than 7 whereas that of the Iranian study used to have a flexible diagnostic criteria of birth asphyxia including: umbilical cord $\mathrm{pH}<7$ or 5 min Apgar score $<6$ or 20 minute Apgar score less than 7 or multi organs failure in the first 72 hours or convulsion in the first 24 hours of life. The prevalence of birth asphyxia in our study was also lower than the study in Nigeria $(21.1 \%)^{19}$ which may be attributed to the use of non APGAR score clinical parameters by the Nigerian study that included even out born neonates. Moreover, the prevalence of birth asphyxia at our study setting was lower than other prior Ethiopian studies at Dilla $(32.8 \%)^{17}$ and Jimma $(47.5 \%)^{51}$ that could be attributed to the relatively smaller sample size in our study and study setting difference (ours is at general hospital, while others were held at referral hospitals where more complicated and referral deliveries present with greater proportion of birth asphyxia than general hospitals)."

In the analysis of associated factors, the likelihood of developing birth asphyxia among neonates born to mothers with antenatal obstetric complications was 2.6 times higher as compared to their counter-parts. Different prior Ethiopian studies ${ }^{17,18,40,41,42}$ revealed similar findings. Besides, the finding was consistent with other studies conducted in both developed and developing countries ${ }^{19,20,43,44,46,49}$ where the odds of birth asphyxia among neonates born to mothers with obstetric complications were about three to eleven times higher than those born to mothers who had no antenatal obstetric complications. This could be due to the fact that antenatal obstetric complications are often accompanied with feto placental hypoperfusion and subsequent ischemia that in turn cause intrauterine fetal hypoxia ${ }^{3,6,52,54}$

Neonates born with fetal malpresentation were 3.2 times more likely of being asphyxiated as compared to those with vertex presentations. This finding was congruent with other Ethiopian studies ${ }^{18,40}$ where the odds of asphyxia neonatorum among the malpresented fetuses were 6.98 times and 4.46 times higher as compared to the vertex presented fetuses respectively. A Cameroonian study ${ }^{20}$ also showed similar finding. This similarity could be due to the fact that malpresentation is often associated with fetal life threatening complications like umbilical cord accidents (cord prolapse, cord compression) and prolonged labor ${ }^{6}$.

Neonates born to mothers with premature rupture of fetal membranes were 6.6 times more prone to be asphyxiated at birth as compared to those with intrapartum rupture. A consistent finding was obtained from studies at Cameroon ${ }^{20}$, Uganda ${ }^{45}$ and Al-Diwaniya teaching hospital ${ }^{48}$. The consistence can be justified by the fact that when fetal membranes rupture prematurely, spontaneous gush of amniotic fluid along with umbilical cord prolapse happens. Moreover, premature rupture of membranes, if prolonged, often facilitates feto-maternal systemic infections ${ }^{3,6,7}$, which is usually ensued by subsequent asphyxia neonatorum.

The risk of birth asphyxia among neonates with history of meconium stained amniotic fluid was 2.7 times higher than those born to mothers with clear amniotic fluid. This finding was consistent with other studies ${ }^{17,18,40-42 \text {, }}$ $45,46,47$. The likely justification may be due to the fact that meconium stained amniotic fluid results in intrapartum inhalation of meconium which leads to mechanical obstruction of airways, surfactant inactivation, chemical inflammation and apoptosis of the pulmonary tissues thereby facilitating pulmonary air leak and hypoxia ${ }^{6,55}$.

From bivariable analysis, it has been shown that neonates born to mothers with birth spacing of $<2$ years were 1.89 times more likely to be asphyxiated as compared to those with $\geq 2$ years of birth spacing. This may be due to the fact that narrow birth spacing ( $<2$ years) is a universal public health problem having association with adverse maternal, fetal and neonatal outcomes such as low birth weight ${ }^{56,57}$, preterm delivery, small for gestational age ${ }^{58}$, precipitous labor ${ }^{59}$, gestational diabetes ${ }^{60,61}$, anemia ${ }^{61,62}$, uterine rupture, premature rupture of membrane, preeclampsia and chronic hypertension 
60, 63, 64. Significance of some of these outcomes is also supported by this study.

\section{Limitation of the study}

The study lacks support of qualitative data. Moreover, the results may not be representative of the entire newborns in Ethiopia due to a small sample size consideration in this study. The study also shares drawbacks of a cross-sectional design.

Despite the aforementioned findings, the results are limited to the diagnosis of birth asphyxia based on solely fifth minute APGAR score. However, to minimize its effect on the validity of our estimate about the prevalence of birth asphyxia, the data collectors underwent efforts of consulting senior professionals (midwives and Integrated Emergency surgery obstetricians) about the neonatal condition to confirm the diagnosis of asphyxia. The study also shares drawbacks of a cross-sectional design. Furthermore, the study lacks support of qualitative data.

\section{Conclusion}

Compared to prior studies at other settings, the prevalence of asphyxia neonatorum in our study area was relatively low. Presence of antenatal obstetric complications, fetal malpresentation, premature rupture of fetal membranes and meconium stained amniotic fluid were significantly associated with increased odds of asphyxia neonatorum. Therefore, early antenatal screening and follow up of every mother should be given due strict emphasis for timely detection and management of any obstetric complication. Besides, mothers with fetal malpresentation should be given thorough partographic follow up of their labor to early detect any fetal abnormality for emergency actions. Mothers with PROM should be provided with prophylactic antibiotics to prevent feto-neonatal systemic infections. Besides, digital vaginal examinations of PROM mothers should be kept as minimal as possible to reduce the likelihood of chorioamnionitis. Appreciating meconium stained amniotic fluid during labor and/delivery should be regarded as a signal of birth asphyxia; hence, delivery care providers have to be ready to give immediate resuscitation for such neonates.

\footnotetext{
Abbreviations

WHO: World Health Organization; ANC: Ante Natal Care; APGAR: Appearance, Pulse Rate, Grimace, Activity, Respiration Rate; CS: Cesarean Section; DTGH: Debre Tabor General Hospital; EDHS: Ethiopian Demographic Health Survey; FMOH: Federal Ministry of
}

Health; GA: Gestational Age; NICU: Neonatal Intensive Care Unit; NMR: Neonatal Mortality Rate; PROM: Premature Rupture of Membrane; SVD: Spontaneous Vaginal Delivery

\section{Declarations}

\section{Ethics approval and consent to participate}

Ethical approval with ethics approval number of DTUCHS-013 was obtained from Debre Tabor University, College of Health Sciences, Institutional Health Research Ethics Review Committee (IHRERC). An informed verbal voluntary consent was obtained from all the eligible mothers. Mothers preferred oral consent because it was a study that they themselves were interested to contribute for the beneficent sake of neonatal health after securing the nonmalficent and confidentiality issues orally as stated in the participant information sheet. Moreover, all eligible mother newborn dyads were further ethically secured by the written, voluntary and signed consent that the authors already made with the hospital ethical review board before the startup of data collection.

\section{Consent to publish \\ Not applicable.}

\section{Availability of data and materials}

Data will be available upon request from the corresponding author.

\section{Competing interests}

The authors declare that they have no competing interests.

\section{Funding}

This research didn't receive any grant from any funding agency in the public, commercial or not-for-profit sectors.

\section{Authors' Contributions}

WA, the corresponding author, worked on designing the study, training and supervising the data collectors, interpreting the result and preparing the manuscript. The co $\neg$ authors namely TG, MY, HS and AY played their roles in analyzing and interpreting the result. All authors were involved in writing, reading and approving of the final manuscript.

\section{Acknowledgments}

The authors acknowledged the director of Debre Tabor General Hospital, data collectors, supervisors and 
data entry operators. The author is also deeply indebted to the Institutional Health Research Ethics Review Committee (IHRERC) of Debre Tabor University for working on the ethical perspectives of the proposal and letting do this study. Last but not least, the respondents deserve the authors' sincerest thanks for their kind responses.

\section{References}

1. Safe Motherhood: Basic newborn resuscitation'. A practical guide. Geneva: World Health Organization; 1997.

2. Birth Asphyxia - Summary of the previous meeting and protocol overview. WHO Geneva Milano, $11^{\text {th }}$ June, 2007 .Accessed 5th March 2019.

3. Lisa M, Cock AD, Lu-Ann Papile. Perinatal Asphyxia. In: John P, Cloherty, Eich enwald, Ann R. Stark. Manual of neonatal care. $6^{\text {th }}$ Ed. Wolters Kluwer, 2008: 518-527.

4. Lisa M, Cock AD, Lu-Ann Papile. Perinatal Asphyxia. In: John P, Cloherty, Eich enwald, Ann R. Stark. Manual of neonatal care. $6^{\text {th }} \mathrm{Ed}$. Wolters Kluwer; 2008: 518-527.

5. Committee on Fetus and Newborn, American Academy of Pediatrics, and Committee on Obstetric Practice, American College of Obstetricians and Gynecologists (1996) American Academy of Pediatrics: Use and Abuse of the Apgar Score. Pediatrics, 98, 141-142.

6. Stoll BJ. Fetal and neonatal infant. In: Behrman RE, Kligman RM, Jenson HB. Nelson: Text book of pediatrics. 18th Ed.; WB. Saunders Company; 2007: 718-720.

7. Federal democratic republic of Ethiopia Ministry of Health, BEmONC LRP (2018): Best practice in maternal and newborn care Maternal Death Surveillance and Response.

8. Haider BA, Bhutta ZA. Birth asphyxia in developing countries: current status and public health implications. Current Problems in Pediatric and Adolescent Health Care. 2006; 5(36):178-188.

9. Ezechukwu CC, Ugochukwu EF, Egbuonu I, Chukwuka JO (2004). Risk factors for neonatal mortality in a regional tertiary hospital in Nigeria. Niger J Clin Pract 7: 50-55.

10. Dilenge, ME, Majnemer A, Shevell MI (2001): Long-Term Developmental Outcome of Asphyxiated Term Neonates. J Child Neurol 16: 781-792.

11. Ruth VJ, Raivio KO (1988) Perinatal brain damage: predictive value of metabolic acidosis and the Apgar score. BMJ 297: 24-27.

12. Ryan S, Gregg T, Patel L (eds) (2003) Core paediatrics: a problem-solving approach. London: Hodder Arnold.
13. Pitsawong C, Panichkul P. Risk factors associated with birth asphyxia in Phramongkutklao Hospital. Thai Journal of Obstetrics and Gynaecology. 2012; 19 (4): 165-171. 14. Ellenberg JH, Nelson KB. The association of cerebral palsy with birth asphyxia: a definitional quagmire. Developmental Medicine \& Child Neurology. 2013; 55(3):210- 216.

15. Halloran DR, McClure E, Chakraborty H, Chomba E, Wright LL. Birth asphyxia survivors in a developing country. Journal of Investigate Medicine. 2006; Vol54(1):309

16. Central Statistical Agency CSA-Ethiopia, and ICF. Ethiopian Demographic and Health Survey: Key Indicators Report. Addis Ababa, Ethiopia and Rockville, Maryland, USA. CSA and ICF: Federal Democratic Republic of Ethiopia; 2016

17. Alemu et al. Pediatric Health, Medicine and Therapeutics. Prevalence and associated factors of perinatal asphyxia among newborns in Dilla University referral hospital, Southern Ethiopia- 2017.

18. Jebessa Wayessa Z, Belachew T, Joseph J. Birth asphyxia and associated factors among newborns delivered in Jimma zone public hospitals, South West Ethiopia: A cross $\neg$ sectional study. Journal of Midwifery and Reproductive Health. 2018; 6(2):1289-1295. DOI:10.22038/ JMRH.2018.10483.

19. Ilah BG, Aminu MS, Musa A, Adelakun MB, Adeniji AO, Kolawole T. Prevalence and risk factors for perinatal asphyxia as seen at a specialist hospital in Gusau, Nigeria. Sub-Saharan African Journal of Medicine 2015, 2(2):64.

20. Chiabi A, Nguefack S, Evelyne M, Nodem S, Mbuagbaw L, Mbonda E, et al. Risk factors for birth asphyxia in an urban health facility in Cameroon. Iranian Journal of Child Neurology 2013, 7(3):46. PMID: 24665306

21. Nayeri F, Shariat M, Dalili H, Adam LB, Mehrjerdi FZ, Shakeri A. Perinatal risk factors for neonatal asphyxia in Vali-e-Asr hospital, Tehran-Iran. Iranian Journal of Reproductive Medicine 2012, 10(2):137. PMID: 25242987.

22. Asad Nauman Kiyani, MBBS; Arshad Khushdil, FCPS; Azra Ehsan, MBBS : Perinatal Factors Leading to Birth Asphyxia among Term Newborns in a Tertiary Care Hospital Department of Pediatrics, Combined Military Hospital Multan, Pakistan. Iran JPediatr, Vol 24 (No 5), Oct 2014.Original article

23. Lawn JE, Cousens S, Zupan J (2005) 4 million neonatal deaths: When? Where? Opportunities for Africa's Newborns Why? Lancet 365: 891-900.

24. Maternal and Newborn Health Disparities in Ethiopia: UNICEF; 2015 
25. Adebami OJ. Maternal and fetal determinants of mortality in babies with birth asphyxia at Osogbo, Southwestern Nigeria. Glo Adv Res J Med Med Sci 2015,

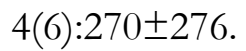

26. Lee AC, Mullany LC, Tielsch JM, Katz J, Khatry SK, LeClerq SC, et al. Risk factors for neonatal mortality due to birth asphyxia in southern Nepal: a prospective, community based cohort study. Pediatrics

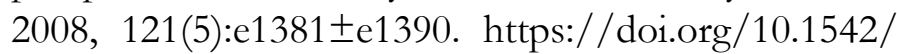
peds.2007-1966: PMID: 18450881

27. Sampa RP, Hossain QZ, Sultana S. Observation of Birth Asphyxia and Its Impact on Neonatal Mortality in Khulna Urban Slum Bangladesh. International Journal of Advanced Nutritional and Health Science 2012, 1(1):pp. $1 \pm 8$.

28. Organization WH. World health statistics 2016: monitoring health for the SDGs sustainable development goals: World Health Organization.

29. Liu L, Oza S, Hogan D, et al. Global, regional and national causes of child mortality in 2000-13, with projections to inform post-2015 priorities: an updated systematic analysis. The Lancet. 2015;385(9966):430-440 30. Becher JC, Stenson B, Lyon A. Is intrapartum asphyxia preventable? BJOG: An International Journal of Obstetrics \& Gynaecology. 2007; 114(11):1442-1444.

31. Kiyani AN, Khushdil A, Ehsan A. Perinatal factors leading to birth asphyxia among term newborns in a tertiary care hospital. Iranian Journal of Pediatrics 2014, 24(5):637. PMID: 25793074

32. De Souza ALS, de Souza NL, de Franqa DF, et al. Risk factors for perinatal asphyxia in newborns delivered at term. Open Journal of Nursing. 2016; 6(07):558.

33. Chandra s, Ramji s, Thirupuram S. Perinatal asphyxia. Multivariate analysis of risk factors in hospital births. Indian pediatr 1997; 34: 206-212 PubMed

34. Berglund S, Grunewald C, Pettersson H, Cnattingius S (2010): Risk factors for asphyxia associated with substandard care during labor. Acta Obstet Gynecol Scand 89: 39-48.

35. Berglund a, Cgrcenewalda, Ratters one, on severe asphyxia due to delivery - related malpractice in Sweden $1990-2005$

36. LF nielsenl, D. shendel 6. On asphyxia related risk factors and their timing in spastics' cerebral palsy. In Denmark, July 2005.

37. Sophie BERGluNDl on risk factors for asphyxia and associated with substandard care during Labor. Acta obstetrician ET Gynacecolagica 2010, 89; 39 - 48

38. Ibrahim NA, Muhye A, Abdulie S (2017): Prevalence of Birth Asphyxia and Associated Factors among
Neonates Delivered in Dilchora Referral Hospital, in Dire Dawa, Eastern Ethiopia. Clinics Mother Child Health 14: 279. doi:10.4172/2090-7214.1000279

39. Miller NP, Amouzou A, Tafesse M, et al. Integrated community case management of childhood illness in Ethiopia: implementation, strength and quality of care. The American Journal of Tropical Medicine and Hygiene. 2014; $91(2): 424$ PubMed -434.

40. Gudayu TW. Proportion and factors associated with low fifth minute Apgar score among singleton newborn babies in Gondar University Referral Hospital; North West Ethiopia. African Health Sciences 2017, 17(1):1 \pm 6. https://doi.org/10.4314/ahs.v17iL2 PMID: 29026371

41. Wosenu L, Worku AG, Teshome DF, Gelagay AA (2018) Determinants of birth asphyxia among live birth newborns in University of Gondar referral hospital, northwest Ethiopia: A case-control study. PLoS One 13(9): e0203763. https://doi.org/ 10.1371/journal. pone. 0203763

42. Tasew et al. BMC Res Notes (2018) 11:496 https:// doi.org/10.1186/s13104-018-3611-3

43. Shireen N, Nahar N, Mollah A. Risk factors and short-term outcome of birth asphyxiated babies in Dhaka Medical College Hospital. Bangladesh Journal of Child Health 2009, 33(3):83 \pm 89 .

44. Torres-Munoz J, Rojas C, Mendoza-Urbano D, MaroAn-Cuero D, Orobio S, EchandoAa C. Risk factors associated with the development of perinatal asphyxia in neonates at the Hospital Universitario del Valle, Cali,

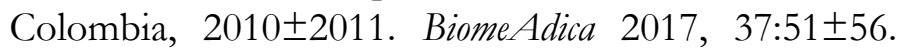
https://doi.org/10.7705/biomedica.v37i1.2844 PMID: 28527266.

45. Kaye D. Antenatal and intrapartum risk factors for birth asphyxia among emergency obstetric referrals in Mulago Hospital, Kampala, Uganda. East African Medi-

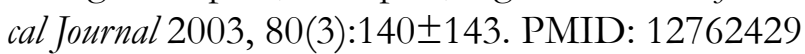

46. Ladfors L, Thiringer K, Niklasson A, Odeback A, Thornberg E. Influence of maternal, obstetric and fetal risk factors on the prevalence of birth asphyxia at term in a Swedish urban population. Acta Obstetricia et

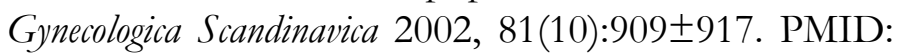
12366480

47. Gane B, Bhat V, Adhisivam B, Joy R, Prasad P, Shruti S. Antenatal and intrapartum risk factors for perinatal asphyxia: A case control study. Current Pediatric Research 2013, 17(2).

48. Sahib HS. Risk factors of perinatal asphyxia: a study at Al-Diwaniya maternity and children teaching

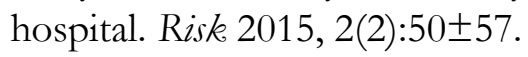


49. Oswyn G, Vince J, Friesen H. Perinatal asphyxia at Port Moresby General Hospital: a study of incidence, risk factors and outcome. Papua New Guinea Medical Jour-

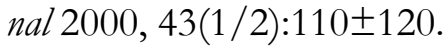

50. Annual report of Debre Tabor General Hospital, 2018.

51. Debelew GT, Afework MF, Yalew AW. Determinants and causes of neonatal mortality in Jimma zone, southwest Ethiopia: a multilevel analysis of prospective follow up study. PLoS One. 2014; 9(9):e107184.

52. Revicky V, Muralidhar A, Mukhopadhyay S, Mahmood T. A case series of uterine rupture: lessons to be learned for future clinical practice. The Journal of

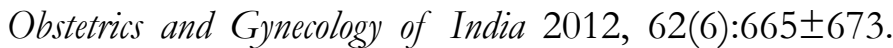
https://doi.org/10.1007/s13224-012-0328-4 PMID: 24293845.

53. South Gondar Zone Health Department: Debre Tabor, 2019.

54. Ethiopian Federal Ministry of Health, 2014. Neonatal Intensive Care Unit (NICU) Training Management Protocol.

55. YurdakoEk M. Meconium aspiration syndrome: do we know? The Turkish Journal of Pediatrics 2011, 53 (2):121. PMID: 21853647.

56. R Thapaliya, BK Rai, R Bhandari, P Rijal and PP Gupta. The effect of birth interval on fetal outcomes. Health Renaissance; 2015; 13(3): 169-76.

57. Mahande M. and Obure J. Effect of interpregnancy interval on adverse pregnancy outcomes in northern Tanzania: a registry-based retrospective cohort study. BMC Pregnancy Childbirth. 2016; 16: 140.
58. Naoko Kozuki, Anne CC Lee, Mariangela F Silveira, et al. The associations of birth intervals with smallfor gestational-age, preterm, and neonatal and infant mortality: a meta-analysis. BMC Public Health. 2013; 13 (Suppl 3):S3 2-9.

59. Appareddy S, Pryor J and Bailey B. Inter-pregnancy interval and adverse outcomes: Evidence for an additional risk in health disparate populations. J Matern Fetal Neonatal Med. 2017; 30: 2640-4.

60. Conde-Agudelo A. , Rosas-Bermudez A. , Fabio Castaño and Maureen $\mathrm{H}$. Norton. Effects of Birth Spacing on Maternal, Perinatal, Infant, and Child Health: A Systematic Review of Causal Mechanisms. Studies in Family Planning 2012; Volume 43 93-114.

61. Hanley G. E., Hutcheon J. A., Kinniburgh B. A. and Lee L. Interpregnancy Interval and Adverse Pregnancy Outcomes: An Analysis of Successive Pregnancies. Obstet Gynecol. 2017; 129: 408-15.

62. Geidam Ad, Inusa A and Isa B. Birth Interval and Maternal Outcome in University Of Maiduguri Teaching Hospital: A Cross Sectional Study. Borno Medical Journal 2016; Vol. 13: issue 2: 132-40.

63. Wendt A, Gibbs CM, Peters S and Hogue CJ. Impact of increasing inter-pregnancy interval on maternal and infant health. Paediatr Perinat Epidemiol. 2012; 26 Suppl 1: 239-58.

64. Bener A. , Mohammed Saleh Najah , Salameh Khalil Mohd Khalil , et al. The impact of the interpregnancy interval on birth weight and other pregnancy outcomes. Rev Bras Saúde Matern Infant, Recife, . 2012; 12 (3): 233-41 